\title{
Three-Dimensional Piecewise-Continuous Class-Shape Transformation of Wings
}

\author{
Erik D. Olson* \\ NASA Langley Research Center, Hampton, VA 23681
}

\begin{abstract}
Class-Shape Transformation (CST) is a popular method for creating analytical representations of the surface coordinates of various components of aerospace vehicles. A wide variety of two- and three-dimensional shapes can be represented analytically using only a modest number of parameters, and the surface representation is smooth and continuous to as fine a degree as desired. This paper expands upon the original two-dimensional representation of airfoils to develop a generalized three-dimensional CST parametrization scheme that is suitable for a wider range of aircraft wings than previous formulations, including wings with significant non-planar shapes such as blended winglets and box wings. The method uses individual functions for the spanwise variation of airfoil shape, chord, thickness, twist, and reference axis coordinates to build up the complete wing shape. An alternative formulation parametrizes the slopes of the reference axis coordinates in order to relate the spanwise variation to the tangents of the sweep and dihedral angles. Also discussed are methods for fitting existing wing surface coordinates, including the use of piecewise equations to handle discontinuities, and mathematical formulations of geometric continuity constraints. A subsonic transport wing model is used as an example problem to illustrate the application of the methodology and to quantify the effects of piecewise representation and curvature constraints.
\end{abstract}

\section{Nomenclature}

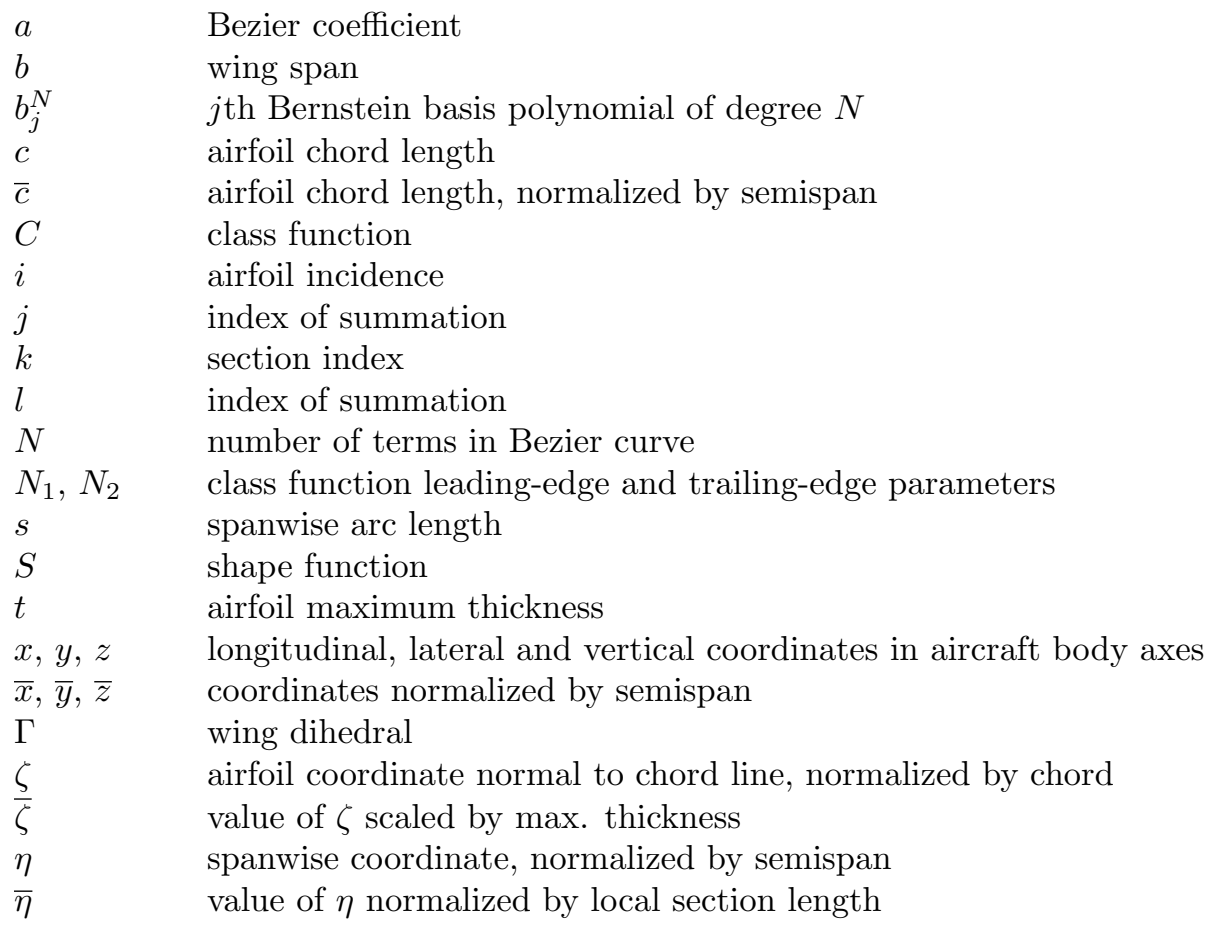

*Aerospace Engineer, NASA Langley Research Center, Senior Member AIAA. 


$\begin{array}{ll}\hat{\eta} & \text { value of } \eta \text { for a known data point } \\ \Lambda_{c / 4} & \text { wing quarter-chord sweep } \\ \chi & \text { generic spanwise parameter } \\ \hat{\chi} & \text { value of } \chi \text { for a known data point } \\ \psi & \text { airfoil chordwise coordinate, normalized by chord } \\ \text { Subscripts } & \\ L & \text { lower surface } \\ \text { root } & \text { value at section root } \\ \text { tip } & \text { value at section tip } \\ T & \text { trailing edge } \\ U & \text { upper surface } \\ \Delta & \text { differential } \\ 0 & \text { reference axis }\end{array}$

\section{Introduction}

7 He Class-Shape Transformation (CST) parametrization method ${ }^{1,2,3}$ has become an increasingly popu-

lar method for creating analytical representations of the surface coordinates of various components of aerospace vehicles. ${ }^{4,5,6,7}$ CST parametrization can be used to represent a wide variety of two- and threedimensional shapes with a modest number of parameters, and it provides built-in design variables for use in shape optimization. The use of analytical functions means that the surface representation is smooth and continuous to as fine a degree as desired, without the need for interpolation between discrete points. The analytical functions also provide the ability to directly compute surface derivatives, normals, and integrals, instead of approximating them using finite-difference methods.

The basic two-dimensional CST methodology describes the normalized surface coordinates of an airfoil at zero incidence using analytical functions of the form $\zeta=\zeta(\psi):(0 \leq \psi \leq 1)$, where $\psi=\left(x-x_{\text {le }}\right) / c$ is the normalized chordwise coordinate and $\zeta=\left(z-z_{\mathrm{le}}\right) / c$ is the coordinate normal to the chord line (Fig. 1). The surface functions take the form of the product of a class function, $C_{N_{2}}^{N_{1}}(\psi)$, and a shape function, $S(\psi)$, with separate functions for each of the upper and lower surfaces:

$$
\begin{array}{ll}
\zeta_{\mathrm{U}}(\psi)= & C_{N_{2}}^{N_{1}}(\psi) S_{\mathrm{U}}(\psi)+\psi \zeta_{T_{U}} \\
\zeta_{\mathrm{L}}(\psi)= & C_{N_{2}}^{N_{1}}(\psi) S_{\mathrm{L}}(\psi)+\psi \zeta_{T_{L}}
\end{array} \mid(0 \leq \psi \leq 1)
$$

The additional $\zeta_{T}$ terms specify the trailing-edge thickness-to-chord ratio for blunt-trailing-edge airfoils. In Eq. 1, the class function, $C_{N_{2}}^{N_{1}}$, takes the form

$$
C_{N_{2}}^{N_{1}}(\psi)=\psi^{N_{1}}(1-\psi)^{N_{2}} \quad(0 \leq \psi \leq 1)
$$

where exponents $N_{1}$ and $N_{2}$ are parameters that define the type of class being represented by the function. Different choices for the values of these parameters can result in a wide range of basic geometry shapes (Table 1). The blunt-nosed airfoil class function $\left(N_{1}=0.5\right.$ and $\left.N_{2}=1\right)$ results in a symmetric airfoil with rounded leading edge and sharp trailing edge that is smooth and continuous everywhere in between (Fig. 2).

In most applications, the shape function takes the form of a Bezier curve of order $N$ :

$$
S(\psi)=\sum_{j=0}^{N} a_{j}\left(\begin{array}{c}
N \\
j
\end{array}\right) \psi^{j}(1-\psi)^{N} \equiv \sum_{j=0}^{N} a_{j} b_{j}^{N}(\psi) \quad(0 \leq \psi \leq 1)
$$

where $\left(\begin{array}{c}N \\ j\end{array}\right)$ is the binomial coefficient and the set $\left\{a_{j}: j=0 \ldots N\right\}$ consists of $N+1$ weighting coefficients for each of the terms in the summation. The weighting coefficients can be used as design variables to modify the shape of the airfoil while maintaining a smooth shape at all times. For an infinite number of terms in the series, the Weierstrass approximation theorem shows that there always exists a set of coefficients that will match any smooth shape exactly. ${ }^{8}$ In addition, if the order of the series is finite, there always exists a sufficiently large number of terms to bound the approximation error within a desired magnitude. A Bezier form of shape function is assumed for all applications in this paper. 


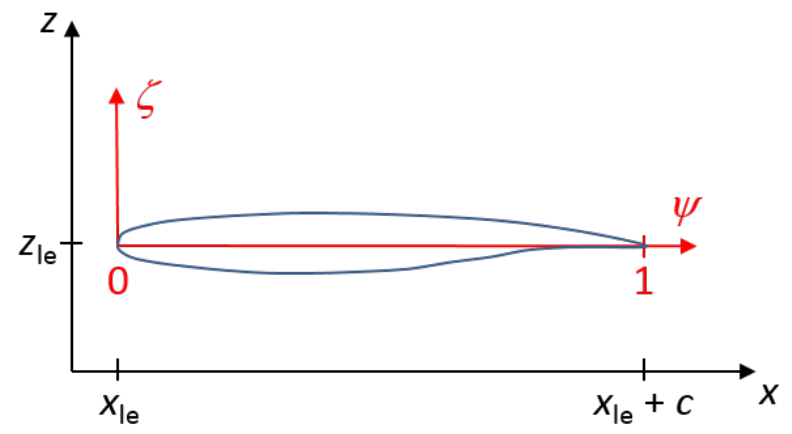

Figure 1. Definition of normalized airfoil coordinates

Table 1. Sample values for coefficients $N_{1}$ and $N_{2}$

\begin{tabular}{|l|l|c|}
\hline$N_{1}$ & $N_{2}$ & Class \\
\hline 0 & 0 & Unit function $^{*}$ \\
0.5 & 1 & Blunt-nosed airfoil \\
0.5 & 0.5 & Elliptical airfoil \\
1 & 0 & Wedge \\
0.75 & 0.75 & Sears-Haack body \\
1 & 1 & Biconvex airfoil \\
\hline
\end{tabular}

* In this paper it is assumed that $0^{0}=1$.

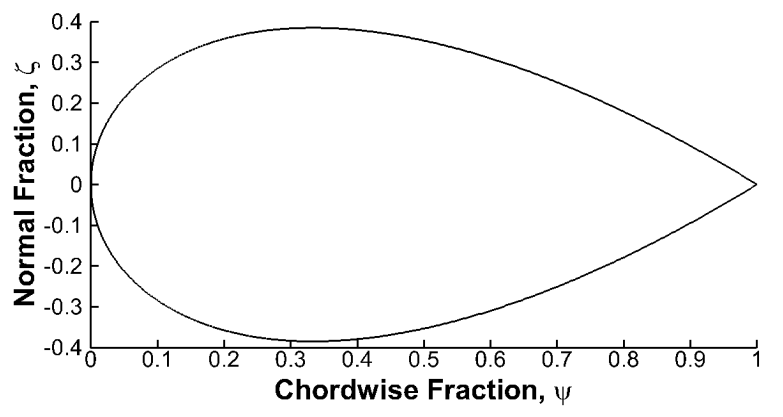

Figure 2. Basic airfoil shape defined by the Kulfan blunt-nosed airfoil class function, $C_{1}^{0.5}$ 
As suggested by Kulfan, ${ }^{2}$ two-dimensional parametrization of the airfoil shape can be extended to three dimensions by representing the wing as an extrusion of parametrized airfoils along a spanwise axis. If one introduces an additional spanwise parameter, $\eta$, where $\eta=0$ represents the root of the wing and $\eta=1$ represents the tip, one can represent the airfoil shape anywhere along the span using an analytical function of the two variables $\psi$ and $\eta$ :

$$
\begin{aligned}
& \bar{\zeta}_{U}(\psi, \eta)=C_{N_{2}}^{N_{1}}(\psi) \sum_{j=0}^{N_{U_{\psi}}} \sum_{l=0}^{N_{U_{\eta}}} a_{U_{j, l}} b_{j}^{N_{U_{\psi}}}(\psi) b_{j}^{N_{U_{\eta}}}(\eta)+\psi \bar{\zeta}_{T_{U}} \\
& \bar{\zeta}_{L}(\psi, \eta)=C_{N_{2}}^{N_{1}}(\psi) \sum_{j=0}^{N_{L_{\psi}}} \sum_{l=0}^{N_{L_{\eta}}} a_{L_{j, l}} b_{j}^{N_{L_{\psi}}}(\psi) b_{j}^{N_{L_{\eta}}}(\eta)+\psi \bar{\zeta}_{T_{L}}
\end{aligned} \mid(0 \leq \psi \leq 1 \cap 0 \leq \eta \leq 1)
$$

Here, the normalized coordinate $\bar{\zeta} \equiv \frac{\zeta}{t / c}$ is used to allow the maximum thickness-to-chord ratio to be scaled independently, as described in Section III. These equations function as a transformation from the non-dimensional $(\psi, \eta)$ space to the physical domain. They are controlled by a set of $N_{U_{\psi}} N_{U_{\eta}}+N_{L_{\psi}} N_{L_{\eta}}$ weighting coefficients to describe the upper- and lower-surface shapes of the airfoils for all locations on the wing.

When the airfoil functions are combined with known spanwise variations of chord, twist, and leadingedge location, the three-dimensional wing surface can be assembled. In several studies, the planform shape has been specified using simple linear parametrizations for a known class of wing (e.g. a simple subsonic transport wing ${ }^{9}$ or a cranked delta wing ${ }^{6}$ ). The planform shape has also been specified by defining the chord and leading-edge $x$ and $z$ coordinates using additional analytical functions of $\eta=2 y / b{ }^{2}$

However, these previous three-dimensional applications lack a methodology of sufficient generality to define all classes of wings, including those with extreme dihedral, such as blended winglets, or those for which the leading-edge $x$ and $z$ coordinates cannot be formulated as monotonic functions of $y$, such as box wings. In addition, many wings exhibit discontinuities in the spanwise variation of parameters, such as chord breaks, so the use of a single, continuous function to describe these parameters can be problematic. This paper proposes a methodology for using spanwise parametrization of the planform shape that retains sufficient generality to be suitable for a much wider range of extruded shapes. Section II formulates the mathematical representation of spanwise-varying parameters, including a method of accounting for discontinuities using piecewise functions in combination with geometric continuity constraints. Section III applies these concepts to formulate a comprehensive representation of general three-dimensional wings. Finally, Section IV applies the methodology to an example problem, that of a subsonic transport wing with a blended winglet.

\section{Spanwise Parametrization}

In addition to three-dimensional parametrization of the airfoils (Eq. 4), this paper introduces a spanwise parametrization scheme for the variation of wing design parameters. For a given parameter, $\chi$, which may be any spanwise-varying property such as chord, twist, etc., one can use CST to create a functional representation of the property between the root and tip of a wing:

$$
\chi(\eta)=C_{N_{2}}^{N_{1}}(\eta) \sum_{j=0}^{N} a_{j} b_{j}^{N}(\eta)+\eta \chi_{\mathrm{tip}}+(1-\eta) \chi_{\mathrm{root}} \quad(0 \leq \eta \leq 1)
$$

The additional term, $(1-\eta) \chi_{\text {root, }}$, accounts for the fact that the property may have a non-zero value at the root, unlike airfoils. The following section examines the process for determining the shape function coefficients that best represent a known spanwise variation.

\section{A. Fitting CST Functions to Existing Shapes}

When using a series representation of a shape such as Eq. 5, the choice of values for the Bezier coefficients to produce a given shape may not be readily obvious. Instead, one would normally start with a given shape and solve for the values of the $N+1$ coefficients $a_{j}, j=0 \ldots N$ which best approximate it. Given $M+3$ ordered pairs of known coordinates $\left(\hat{\eta}_{j}, \hat{\chi}_{j}\right), j=0 \ldots M+2$, with $\hat{\eta}_{0}=0$ and $\hat{\eta}_{M+2}=1$, one can insert the values of $\hat{\eta}_{j}$ and $\hat{\chi}_{j}$ in turn into Eq. 5 and create a system of linear equations. Since, by identity, $\chi_{\text {root }}=\hat{\chi}_{0}$ 
and $\chi_{\text {tip }}=\hat{\chi}_{M+2}$, those terms can be moved to the right side of the equation, and the set of equations for each of the interior points gives $M+1$ equations for $N+1$ variables:

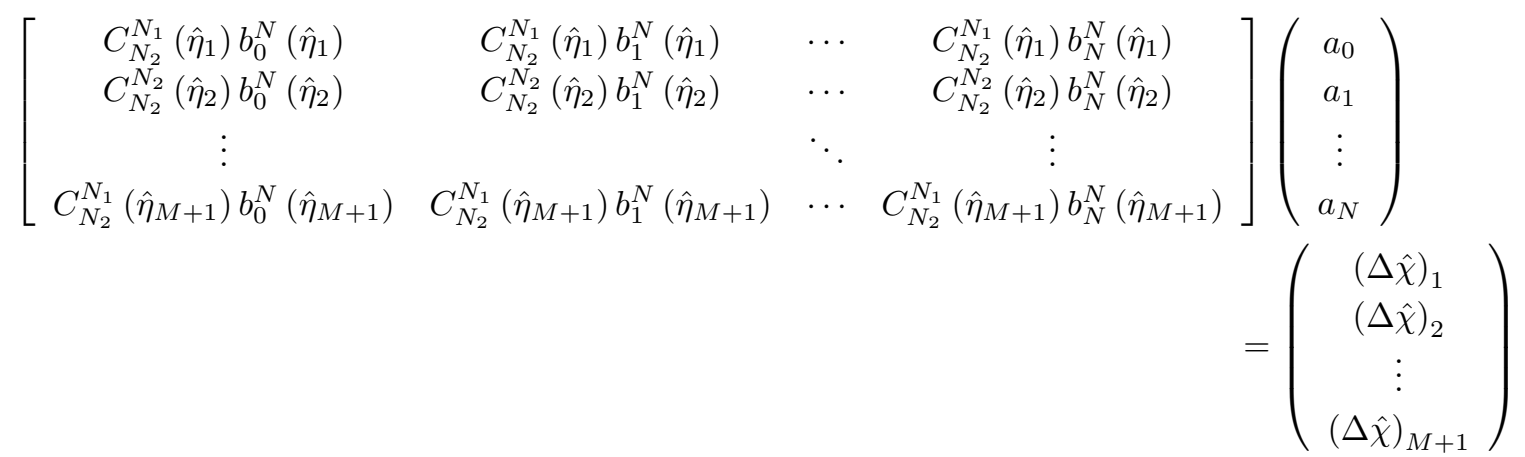

where $(\Delta \hat{\chi})_{j} \equiv \hat{\chi}_{j}-\hat{\eta}_{j} \hat{\chi}_{M+2}-\left(1-\hat{\eta}_{j}\right) \hat{\chi}_{0}$.

If the number of interior points to fit is equal to the number of coefficients $(M=N)$, the exact solution for the coefficients $a_{j}$ can be found through direct matrix inversion or a comparable iterative method. If the number of points is greater than the number of coefficients $(M>N)$, the coefficients can instead be approximated through a pseudo-inverse solution (least-squares fit).

If the shape to be approximated is very non-linear or the interval length between points varies significantly, using an exact solution to Eq. 6 can result in over-fitting of the points and the introduction of spurious oscillations in the intervals between the known points; using a least-squares fit in these cases can help alleviate these problems. In general, experience has shown that it is best to use a number of known points that is two to three times larger than the number of coefficients to be estimated.

Figure 3(a) gives an example of a shape that is particularly challenging: the leftmost and rightmost sections of the shape are purely linear, with a rapid non-linear change in $\chi$ in the middle section $(0.4<\eta<$ $0.6)$. Using an exact solution $(M=N=16)$ results in a function that does indeed pass through all the data points, but its behavior is significantly different than the underlying function from which the data points were sampled, particularly in the linear regions. Doubling the number of data points $(M=35)$, for the same number of coefficients, greatly improves the stability of the fit. Note, however, that with $M>N$, the CST function only approximates the data points, which is especially obvious at $\eta=0.4$ and $\eta=0.6$.

\section{B. Piecewise Representation of Complex Shapes}

The use of CST functions to approximate shapes that do not deviate significantly from their class function usually produces acceptable results. In complex cases like the one shown previously in Fig. 3(a), however, a large number of coefficients and correspondingly large number of sampled data points may be required to reduce the amplitude of the oscillations between known points to an acceptable level. As an alternative, the shape may be represented in a piecewise manner by defining functions on two or more adjoining sub-intervals and using an appropriate number of Bezier coefficients for each. If the curve is divided at $P+2$ break points $\eta_{0}, \eta_{1}, \eta_{2}, \ldots \eta_{P+1}$, where $\eta_{0}=0$ and $\eta_{P+1}=1$, then the equation for the generic parameter $\chi$ becomes a combination of the equations over the $P+1$ intervals:

$$
\chi(\eta)=\left\{\begin{array}{ccc}
C_{N_{2}}^{N_{1}}\left(\bar{\eta}_{0}\right) \sum_{j=0}^{N_{0}} a_{j_{0}} b_{j}^{N_{0}}\left(\bar{\eta}_{0}\right)+\bar{\eta}_{0} \chi_{\mathrm{tip}_{0}}+\left(1-\bar{\eta}_{0}\right) \chi_{\text {ooot }_{0}} & \left(0 \leq \eta<\eta_{1}\right) \\
C_{N_{2}}^{N_{1}}\left(\bar{\eta}_{1}\right) \sum_{j=0}^{N_{1}} a_{j_{1}} b_{j}^{N_{1}}\left(\bar{\eta}_{1}\right)+\bar{\eta}_{1} \chi_{\mathrm{tip}_{1}}+\left(1-\bar{\eta}_{1}\right) \chi_{\operatorname{root}_{1}} & \left(\eta_{1} \leq \eta<\eta_{2}\right) \\
\vdots & \\
C_{N_{2}}^{N_{1}}\left(\bar{\eta}_{P}\right) \sum_{j=0}^{N_{P}} a_{j_{P}} b_{j}^{N_{P}}\left(\bar{\eta}_{P}\right)+\bar{\eta}_{P} \chi_{\mathrm{tip}_{P}}+\left(1-\bar{\eta}_{P}\right) \chi_{\operatorname{root}_{P}} & \left(\eta_{P} \leq \eta \leq 1\right)
\end{array}\right.
$$

where $\bar{\eta}_{k} \equiv \frac{\eta-\eta_{k}}{\eta_{k+1}-\eta_{k}}$.

Using this piecewise definition of the spanwise function, Eq. 6 may be modified to create a block diagonal matrix equation. In this case there are $P+1$ sets of ordered pairs, with the $k$ th set $(0 \leq k \leq P)$ having $M_{k}+3$ ordered pairs of coordinates $\left(\hat{\eta}_{j_{k}}, \hat{\chi}_{j_{k}}\right), j=0 \ldots M_{k}+2$, with $\hat{\eta}_{0_{k}}=\eta_{k}$ and $\hat{\eta}_{M_{k}+2_{k}}=\eta_{k+1}$. Again, by 


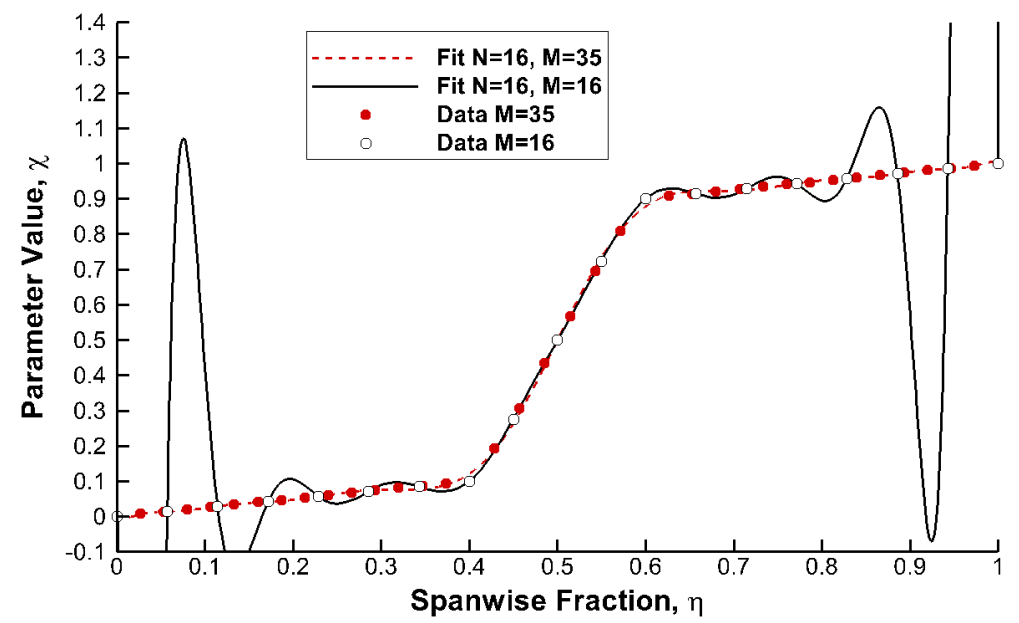

(a) Single-section fit with two data set sizes

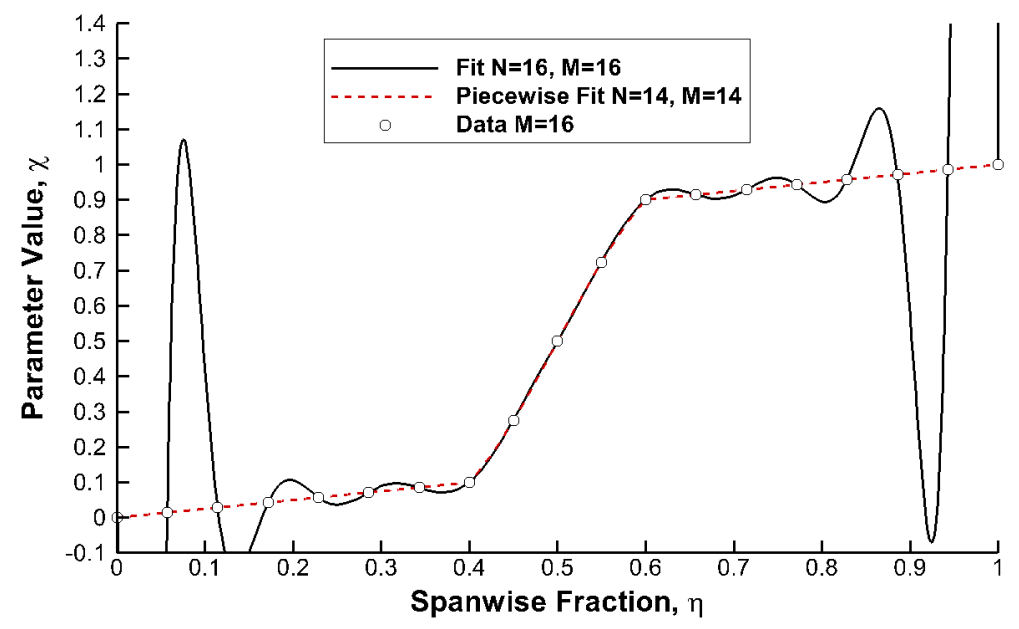

(b) Single-section vs. piecewise fit

Figure 3. Sample CST curve fits using a $C_{1}^{1}$ class function and Bezier-polynomial shape function(s) with 16 coefficients total 
identity $\chi_{\operatorname{root}_{k}}=\hat{\chi}_{0_{k}}$ and $\chi_{\operatorname{tip}_{k}}=\hat{\chi}_{M+2_{k}}$, so the system consists of $\sum_{k=0}^{P}\left(M_{k}+1\right)$ equations for $\sum_{k=0}^{P}\left(N_{k}+1\right)$ variables:

$$
\left[\begin{array}{cccc}
{\left[C_{0}\right]} & 0 & \cdots & 0 \\
0 & {\left[C_{1}\right]} & \cdots & 0 \\
\vdots & \vdots & \ddots & \vdots \\
0 & 0 & \cdots & {\left[C_{P}\right]}
\end{array}\right]\left(\begin{array}{c}
{\left[a_{0}\right]} \\
{\left[a_{1}\right]} \\
\vdots \\
{\left[a_{P}\right]}
\end{array}\right)=\left(\begin{array}{c}
{\left[\chi_{0}\right]} \\
{\left[\chi_{1}\right]} \\
\vdots \\
{\left[\chi_{P}\right]}
\end{array}\right)
$$

where

$$
\begin{gathered}
{\left[C_{k}\right]=\left[\begin{array}{ccc}
C_{N_{2}}^{N_{1}}\left(\overline{\hat{\eta}}_{1_{k}}\right) b_{0}^{N_{k}}\left(\overline{\hat{\eta}}_{1_{k}}\right) & \cdots & C_{N_{2}}^{N_{1}}\left(\overline{\hat{\eta}}_{1_{k}}\right) b_{N_{k}}^{N_{k}}\left(\overline{\hat{\eta}}_{1_{k}}\right) \\
\vdots & \ddots & \vdots \\
C_{N_{2}}^{N_{1}}\left(\hat{\hat{\eta}}_{M_{k}+1_{k}}\right) b_{0}^{N_{k}}\left(\overline{\hat{\eta}}_{M_{k}+1_{k}}\right) \cdots & C_{N_{2}}^{N_{1}}\left(\overline{\hat{\eta}}_{M_{k}+1_{k}}\right) b_{N_{k}}^{N_{k}}\left(\overline{\hat{\eta}}_{M_{k}+1_{k}}\right)
\end{array}\right]} \\
{\left[a_{k}\right]=\left(\begin{array}{c}
a_{0_{k}} \\
a_{1_{k}} \\
\vdots \\
a_{N_{k}}
\end{array}\right)} \\
{\left[\begin{array}{c}
(\Delta \hat{\chi})_{1_{k}} \\
(\Delta \hat{\chi})_{2_{k}} \\
\vdots \\
(\Delta \hat{\chi})_{M_{k}+1_{k}}
\end{array}\right)}
\end{gathered}
$$

while $\overline{\hat{\eta}}_{j_{k}} \equiv \frac{\hat{\eta}_{j_{k}}-\eta_{k}}{\eta_{k+1}-\eta_{k}}$ and $(\Delta \hat{\chi})_{j_{k}} \equiv \hat{\chi}_{j_{k}}-\overline{\hat{\eta}}_{j_{k}} \hat{\chi}_{M+2_{k}}-\left(1-\overline{\hat{\eta}}_{j_{k}}\right) \hat{\chi}_{0_{k}}$.

Figure 3(b) illustrates how fitting of the previous example can be greatly improved through the use of piecewise functions. For the piecewise case, the number of interior data points available for curve fitting is reduced by two because the data points at $\eta=0.4$ and $\eta=0.6$ now become end points for multiple segments; additionally, the number of Bezier coefficients is reduced by two because of the need to specify the function values at the joints $\left(\chi_{\mathrm{tip}_{0}}=\chi_{\mathrm{root}_{1}}=0\right.$ and $\left.\chi_{\mathrm{tip}_{1}}=\chi_{\operatorname{root}_{2}}=1\right)$. Nevertheless, the piecewise parametrization scheme exhibits improved performance for the same number of total data points and fitting coefficients. All of the Bezier coefficients are used for the middle segment, while none are required for the left and right segments since those regions are linear.

\section{Geometric Continuity Constraints}

The single-section parametrization scheme of Eq. 5 is continuous, as are all of its derivatives. However, a piecewise representation generally is not continuous across the section breaks, and its use may introduce discontinuities even when the sampled data points come from a continuous curve. There are cases in which it is desirable for the shape to exhibit a certain degree of continuity in order to produce acceptable performance, such as low drag, resistance to boundary-layer separation, etc. If two curves are joined, they are said to have zero-order geometric continuity, $G^{0}$, at the joint if their end points exactly coincide. ${ }^{10}$ In addition, the curves have first-order geometric continuity, $G^{1}$, if their tangents point in the same direction at the joint; and they have second-order geometric continuity, $G^{2}$, if their centers of curvature at the joint coincide. In the following sections, the conditions for enforcing geometric zero-, first- and second-order continuity across section breaks of a piecewise parametrization scheme are derived. The continuity conditions are substituted into Eq. 8 to derive a modified form of the linear set of equations.

The behavior of the class function, $C_{N_{2}}^{N_{1}}(\eta)$, at its end points $(\eta=0$ and $\eta=1)$ can vary greatly depending on the values of $N_{1}$ and $N_{2}$; the behavior at the end points in turn affects the form of the continuity constraint equations. For the biconvex class function, $C_{1}^{1}(\eta)$, the values of the function and its derivatives at the end 
points are

$$
\begin{aligned}
& C_{1}^{1}(0)=\quad 0, \quad C_{1}^{1}(1)=0 \\
& C^{\prime 1}{ }_{1}^{1}(0)=\quad 1, \quad C^{\prime 1}{ }_{1}(1)=-1 \\
& C^{\prime \prime}{ }_{1}^{1}(0)=-2, \quad C^{\prime \prime 1}{ }_{1}^{1}(1)=-2
\end{aligned}
$$

whereas for the the unit class function, $C_{0}^{0}(\eta)$, the values of the function and its derivatives at the end points are

$$
\begin{aligned}
C_{0}^{0}(0) & =1, \quad C_{0}^{0}(1)=1 \\
C^{\prime}{ }_{0}^{0}(0) & =0, \quad C_{0}^{\prime 0}(1)=0 \\
C^{\prime \prime \prime}{ }_{0}^{0}(0) & =0, \quad C^{\prime \prime 0}{ }_{0}^{0}(1)=0
\end{aligned}
$$

The following discussion is limited to the derivation of continuity constraints for these two forms of class function, in addition to the blunt-nosed airfoil class function, $C_{1}^{0.5}(\eta)$.

\section{Zero-Order Continuity}

For the piecewise parametrization of generic parameter $\chi$ (Eq. 7), the values of the function at the root and tip of section $k$ are

$$
\begin{aligned}
\chi_{k}\left(\eta_{k}\right) & =C_{N_{2}}^{N_{1}}(0) a_{0_{k}}+\chi_{\operatorname{root}_{k}} \\
\chi_{k}\left(\eta_{k+1}\right) & =C_{N_{2}}^{N_{1}}(1) a_{N_{k}}+\chi_{\operatorname{tip}_{k}}
\end{aligned}
$$

If the tip of segment $k$ is joined to the root of segment $k+1$, enforcing $G^{0}$ continuity at the joint requires that $\chi_{k}\left(\eta_{k+1}\right)=\chi_{k+1}\left(\eta_{k+1}\right)$. For the biconvex class function, the value is zero at the end points so ensuring $G^{0}$ continuity requires only that

$$
\chi_{\text {root }_{k+1}}=\chi_{\operatorname{tip}_{k}}
$$

For a pure Bezier parametrization, there is an additional requirement that

$$
a_{0_{k+1}}=a_{N_{k}}
$$

\section{First-Order Continuity}

The equations for the derivative of the CST parametrization of section $k$ at the root and tip are

$$
\begin{array}{rlrr}
\chi_{k}^{\prime}\left(\eta_{k}\right) & =C_{N_{2}}^{\prime N_{1}}(0) a_{0_{k}}+ & N_{k} C_{N_{2}}^{N_{1}}(0)\left(-a_{0_{k}}+a_{1_{k}}\right)+\Delta \chi_{k} \\
\chi_{k}^{\prime}\left(\eta_{k+1}\right) & =C_{N_{2}}^{N_{1}}(1) a_{N_{k}}+ & N_{k} C_{N_{2}}^{N_{1}}(1)\left(-a_{N-1_{k}}+a_{N_{k}}\right)+\Delta \chi_{k}
\end{array}
$$

where $\Delta \chi_{k} \equiv \chi_{\operatorname{tip}_{k}}-\chi_{\text {root }_{k}}$. For $G^{1}$ continuity at the joint between sections $k$ and $k+1$, the slopes of the two curves must be equal at that point:

$$
\frac{\chi_{k}^{\prime}\left(\eta_{k+1}\right)}{\Delta \eta_{k}}=\frac{\chi_{k+1}^{\prime}\left(\eta_{k+1}\right)}{\Delta \eta_{k+1}}
$$

where $\Delta \eta_{k} \equiv\left(\eta_{k+1}-\eta_{k}\right)$. For the biconvex class function, one can combine the $G^{0}$ continuity condition (Eq. 12) with Eqns. 9, 14, and 15, then solve for $a_{0_{k+1}}$ as a function of $a_{N_{k}}$ :

$$
a_{0_{k+1}}=-\frac{\Delta \eta_{k+1}}{\Delta \eta_{k}} a_{N_{k}}+\frac{\Delta \eta_{k+1}}{\Delta \eta_{k}} \Delta \chi_{k}-\Delta \chi_{k+1}
$$

For a pure Bezier parametrization, one instead uses Eqns. 10, 13, 14, and 15 to solve for $a_{1_{k+1}}$ as a function of $a_{N_{k}}$ and $a_{N-1_{k}}$ :

$$
a_{1_{k+1}}=-\frac{\Delta \eta_{k+1}}{\Delta \eta_{k}} \frac{N_{k}}{N_{k+1}} a_{N-1_{k}}+\left(1+\frac{\Delta \eta_{k+1}}{\Delta \eta_{k}} \frac{N_{k}}{N_{k+1}}\right) a_{N_{k}}+\frac{1}{N_{k+1}}\left(\frac{\Delta \eta_{k+1}}{\Delta \eta_{k}} \Delta \chi_{k}-\Delta \chi_{k+1}\right)
$$

For a blunt-nosed airfoil $\left(N_{1}=0.5\right)$, the derivatives of the upper- and lower-surface functions are positive and negative infinity, respectively, at the leading edge, so first-order continuity is automatically maintained between the upper and lower surfaces. 


\section{Second-Order Continuity}

The equations for the second derivative of the CST parametrization of section $k$ at the root and tip are

$$
\begin{aligned}
\chi_{k}^{\prime \prime}\left(\eta_{k}\right)= & C^{\prime \prime}{ }_{N_{2}}^{N_{1}}(0) a_{0_{k}}+2 N_{k}{C^{\prime}}_{N_{2}}^{N_{1}}(0)\left(-a_{0_{k}}+a_{1_{k}}\right)+ \\
& N_{k}\left(N_{k}-1\right) C_{N_{2}}^{N_{1}}(0)\left(a_{0_{k}}-2 a_{1_{k}}+a_{2_{k}}\right) \\
\chi_{k}^{\prime \prime}\left(\eta_{k+1}\right)= & C^{\prime \prime N_{1}}(1) a_{N_{k}}+2 N_{k} C_{N_{2}}^{\prime N_{1}}(1)\left(-a_{N-1_{k}}+a_{N_{k}}\right)+ \\
& N_{k}\left(N_{k}-1\right) C_{N_{2}}^{N_{1}}(1)\left(a_{N-2_{k}}-2 a_{N-1_{k}}+a_{N_{k}}\right)
\end{aligned}
$$

For $G^{2}$ continuity at the joint between sections $k$ and $k+1$, the centers of curvature for the two curves must be the same. As a necessary condition, the radii of curvature of the two curves must be equal, and since it is already established from $G^{1}$ continuity that the magnitudes of the first derivatives are equal, this means that the magnitude of the second derivatives of the two curves must also be equal at the joint:

$$
\left|\frac{\chi_{k}^{\prime \prime}\left(\eta_{k+1}\right)}{\left(\Delta \eta_{k}\right)^{2}}\right|=\left|\frac{\chi_{k+1}^{\prime \prime}\left(\eta_{k+1}\right)}{\left(\Delta \eta_{k+1}\right)^{2}}\right|
$$

For section $k$ connected at the tip to section $k+1$ at the root, the sign of the second derivatives must be the same. For two sections connected at their roots (such as two airfoil surfaces connected at the leading edge), the second derivatives would be of equal magnitude but opposite sign.

For the biconvex class function, one can combine the $G^{1}$ continuity condition (Eq. 16) with Eqns. 9, 18, and 19, then solve for $a_{1_{k+1}}$ :

$$
\begin{aligned}
a_{1_{k+1}}= & \left(\frac{\Delta \eta_{k+1}}{\Delta \eta_{k}}\right)^{2} \frac{N_{k}}{N_{k+1}} a_{N-1_{k}}-\left[\left(\frac{\Delta \eta_{k+1}}{\Delta \eta_{k}}\right)^{2} \frac{N_{k}+1}{N_{k+1}}+\frac{\Delta \eta_{k+1}}{\Delta \eta_{k}} \frac{N_{k+1}+1}{N_{k+1}}\right] a_{N_{k}}+ \\
& \frac{N_{k+1}+1}{N_{k+1}}\left(\frac{\Delta \eta_{k+1}}{\Delta \eta_{k}} \Delta \chi_{k}-\Delta \chi_{k+1}\right)
\end{aligned}
$$

For a pure Bezier parametrization, one instead uses Eqns. 10, 13, 17, 18, and 19 to solve for $a_{2_{k+1}}$ :

$$
\begin{aligned}
a_{2_{k+1}}= & \left(\frac{\Delta \eta_{k+1}}{\Delta \eta_{k}}\right)^{2} \frac{N_{k}}{N_{k+1}} \frac{N_{k}-1}{N_{k+1}-1} a_{N-2_{k}}-2 \frac{\Delta \eta_{k+1}}{\Delta \eta_{k}} \frac{N_{k}}{N_{k+1}}\left[1+\frac{\Delta \eta_{k+1}}{\Delta \eta_{k}} \frac{N_{k}-1}{N_{k+1}-1}\right] a_{N-1_{k}}+ \\
& {\left[\left(\frac{\Delta \eta_{k+1}}{\Delta \eta_{k}}\right)^{2} \frac{N_{k}}{N_{k+1}} \frac{N_{k}-1}{N_{k+1}-1}+2 \frac{\Delta \eta_{k+1}}{\Delta \eta_{k}} \frac{N_{k}}{N_{k+1}}+1\right] a_{N_{k}}+\frac{2}{N_{k+1}}\left(\frac{\Delta \eta_{k+1}}{\Delta \eta_{k}} \Delta \chi_{k}-\Delta \chi_{k+1}\right) }
\end{aligned}
$$

For the the blunt-nosed airfoil class, the second derivative of both the upper- and lower-surface curves is infinite at the leading edge, but Kulfan ${ }^{1}$ has shown that the radius of curvature is finite and that $G^{2}$ continuity can be enforced simply by setting $a_{0_{k+1}}=-a_{0_{k}}$.

\section{CST Parametrization of an Aircraft Wing}

This section derives a complete set of functions to represent a wing using CST parametrization in the form of Eq. 5. One can define a reference axis, which is the locus of the quarter-chord points of the airfoils from root $(\eta=0)$ to tip $(\eta=1)$. The coordinates along this axis are $\left(x_{0}, y_{0}, z_{0}\right)$, and the spanwise distance, $s$, is the arc length along a projection of the reference axis onto the $x=0$ plane:

$$
s(\eta)=\int_{0}^{\eta} \sqrt{y_{0}^{2}+z_{0}^{2}} \mathrm{~d} \eta
$$

The reference span, $b$, is twice the spanwise distance at the tip $\left(\left.b \equiv 2 s\right|_{\eta=1}\right)$.

If one considers the wing to be a continuous extrusion of airfoils, one can define parametrizations that define the variation of chord, $c$, thickness-to-chord ratio, $t / c$, and incidence, $i$, of the airfoil along the reference 
axis:

$$
\begin{array}{llll}
\bar{c}(\eta) & =C_{N_{2}}^{N_{1}}(\eta) \sum_{j=0}^{N_{c}} a_{c_{j}} b_{j}^{N_{c}}(\eta)+ & \eta \bar{c}_{\text {tip }}+ & (1-\eta) \bar{c}_{\text {root }} \\
\frac{t}{c}(\eta) & =C_{N_{2}}^{N_{1}}(\eta) \sum_{j=0}^{N_{t}} a_{t_{j}} b_{j}^{N_{t}}(\eta)+ & \eta\left(\frac{t}{c}\right)_{\text {tip }}+ & (1-\eta)\left(\frac{t}{c}\right)_{\text {root }} \\
i(\eta) & =C_{N_{2}}^{N_{1}}(\eta) \sum_{j=0}^{N_{i}} a_{i_{j}} b_{j}^{N_{i}}(\eta)+ & \eta i_{\text {tip }}+ & (1-\eta) i_{\text {root }}
\end{array} \mid
$$

where $\bar{c} \equiv \frac{2 c}{b}$. These quantities are familiar to the aircraft designer and their spanwise variations follow directly from non-dimensional wing design parameters such as aspect ratio, taper ratio, washout, etc. They represent scaling and rotation operations applied to the normalized airfoil surface obtained from Eqs. 4. Any or all of the sets of Bezier coefficients $\left(\left\{a_{c_{j}}\right\},\left\{a_{t_{j}}\right\}\right.$ or $\left.\left\{a_{i_{j}}\right\}\right)$ may be used as design variables in shape optimization studies. Using separate equations for these parameters means that an optimization study could be performed on one of the quantities of interest independently; for example, the coefficients of the incidence equation could be chosen to match a desired spanwise lift distribution, all while keeping the airfoil, chord, and thickness distributions constant.

One or more of these parametrizations could also be formulated as piecewise equations in the form of Eq. 7, with or without continuity constraints. The form of each equation can be specified separately, so that the chord equation might be defined in a piecewise manner with first-order discontinuity at a chord break; whereas the thickness and incidence equations might, at the same time, be defined as continuous across the break, or even as single-section parametrizations across the entire span. The use of separate equations give the designer a great deal of flexibility to choose the best form of equation for each parameter.

\section{A. Parametrization of Reference Axis Coordinates}

In addition to the distribution of the chord, thickness, and incidence, one also needs to know the physical locations of the airfoils along the span before one can assemble the full wing shape. One method of specifying these reference coordinates is to parametrize them directly using separate functions of $\eta$, as follows:

$$
\begin{aligned}
& \bar{x}_{0}(\eta)=C_{N_{2}}^{N_{1}}(\eta) \sum_{j=0}^{N_{x}} a_{x_{j}} b_{j}^{N_{x}}(\eta)+\eta \bar{x}_{\text {tip }}+(1-\eta) \bar{x}_{\text {root }} \\
& \bar{y}_{0}(\eta)=C_{N_{2}}^{N_{1}}(\eta) \sum_{j=0}^{N_{y}} a_{y_{j}} b_{j}^{N_{y}}(\eta)+\eta \bar{y}_{\text {tip }}+(1-\eta) \bar{y}_{\text {root }} \mid \\
& \bar{z}_{0}(\eta)=C_{N_{2}}^{N_{1}}(\eta) \sum_{j=0}^{N_{z}} a_{z_{j}} b_{j}^{N_{z}}(\eta)+\eta \bar{z}_{\text {tip }}+(1-\eta) \bar{z}_{\text {root }}
\end{aligned} \mid
$$

where $\bar{x}_{0} \equiv \frac{2 x_{0}}{b}, \bar{y}_{0} \equiv \frac{2 y_{0}}{b}$, and $\bar{z}_{0} \equiv \frac{2 z_{0}}{b}$. This direct parametrization scheme is simple and straightforward; on the other hand, the functions themselves may not offer insight to the aircraft designer, who is used to working with such wing shape parameters as sweep and dihedral.

It is apparent that the quarter-chord sweep, $\Lambda_{\frac{c}{4}}$, and dihedral, $\Gamma$, are related to the derivatives of the reference axis coordinates as follows:

$$
\begin{aligned}
\Lambda_{\frac{c}{4}} & =\tan ^{-1}\left(\frac{\mathrm{d} x_{0}}{\mathrm{~d} s}\right) \\
\Gamma & =\tan ^{-1}\left(\frac{\mathrm{d} z_{0}}{\mathrm{~d} y_{0}}\right)=\tan ^{-1}\left(\frac{\mathrm{d} z_{0} / \mathrm{d} s}{\mathrm{~d} y_{0} / \mathrm{d} s}\right)
\end{aligned}
$$

Therefore, it may be preferable to use an alternative form of the reference axis equations, written in terms 
of the derivatives:

$$
\begin{array}{lll}
\left(\frac{\mathrm{d} x_{0}}{\mathrm{~d} s}\right)(\eta)=C_{N_{2}}^{N_{1}}(\eta) \sum_{j=0}^{N_{x}} a_{x_{j}} b_{j}^{N_{x}}(\eta) & +\eta\left(\frac{\mathrm{d} x_{0}}{\mathrm{~d} s}\right)_{\text {tip }} & +(1-\eta)\left(\frac{\mathrm{d} x_{0}}{\mathrm{~d} s}\right)_{\text {root }} \\
\left(\frac{\mathrm{d} y_{0}}{\mathrm{~d} s}\right)(\eta)=C_{N_{2}}^{N_{1}}(\eta) \sum_{j=0}^{N_{y}} a_{y_{j}} b_{j}^{N_{y}}(\eta) & +\eta\left(\frac{\mathrm{d} y_{0}}{\mathrm{~d} s}\right)_{\text {tip }} & +(1-\eta)\left(\frac{\mathrm{d} y_{0}}{\mathrm{~d} s}\right)_{\operatorname{root}} \\
\left(\frac{\mathrm{d} z_{0}}{\mathrm{~d} s}\right)(\eta)=C_{N_{2}}^{N_{1}}(\eta) \sum_{j=0}^{N_{z}} a_{z_{j}} b_{j}^{N_{z}}(\eta) & +\eta\left(\frac{\mathrm{d} z_{0}}{\mathrm{~d} s}\right)_{\text {tip }} & +(1-\eta)\left(\frac{\mathrm{d} z_{0}}{\mathrm{~d} s}\right)_{\operatorname{root}}
\end{array} \mid(0 \leq \eta)
$$

In this alternative form, the reference axis is derived by integrating the derivative equations in the spanwise direction:

$$
\begin{aligned}
& \bar{x}_{0}(\eta)=\int_{0}^{\eta}\left(\frac{\mathrm{d} x_{0}}{\mathrm{~d} s}\right) \mathrm{d} \eta \\
& \bar{y}_{0}(\eta)=\int_{0}^{\eta}\left(\frac{\mathrm{d} y_{0}}{\mathrm{~d} s}\right) \mathrm{d} \eta \\
& \bar{z}_{0}(\eta)=\int_{0}^{\eta}\left(\frac{\mathrm{d} z_{0}}{\mathrm{~d} s}\right) \mathrm{d} \eta
\end{aligned}
$$

Marshall ${ }^{11}$ has shown that CST parametrizations of all class functions with integer class parameters $N_{1}$ and $N_{2}$, as well as the blunt-nosed airfoil class, can be expressed as pure Bezier curves; and since Bezier curves have closed-form integrals, ${ }^{8}$ this means that the integrals for these classes retain the analytical nature of the CST parametrization scheme.

Although they are only directly related to the tangents of the sweep and dihedral, the alternative formulation of Eq. 26 nonetheless can be a more intuitive form for the aircraft designer. In addition, the values for the sweep and dihedral are often constant across the span so these equations can be simple in their form.

Depending on the needs of the designer, either Eqs. 24 or Eqs. 26 may be used to define the coordinates of the reference axis.

\section{B. Assembly of the Three-Dimensional Wing Surface}

Using the full suite of equations (Eqs. 4 and 23), plus either Eqs. 24 or 26, the three-dimensional wing surface is assembled as follows:

1. For any given point in untransformed space, $(\psi, \eta)$, the normalized coordinates of the airfoil upper and lower surfaces are determined from $\bar{\zeta}_{U}(\psi, \eta)$ and $\bar{\zeta}_{L}(\psi, \eta)$.

2. The chordwise coordinate of the airfoil is scaled by $\frac{b}{2} c(\eta)$ and the normal coordinate is scaled by $\frac{b}{2} c(\eta) \frac{t}{c}(\eta)$

3. The normalized coordinates of the spanwise reference point $\left(\bar{x}_{0}, \bar{y}_{0}, \bar{z}_{0}\right)$ are determined and scaled by $\frac{b}{2}$, and the dihedral of the section is determined from the derivatives of the spanwise equations using the second of Eqs. 25.

4. The scaled section is rotated about its quarter-chord by the incidence angle and then about the $x$-axis by the dihedral angle, and finally it is translated so that its quarter-chord point coincides with the reference point. 
In matrix notation, the above transformation steps can be expressed as

$$
\begin{aligned}
\left(\begin{array}{l}
x \\
y \\
z
\end{array}\right) & =\frac{b}{2}\left(\begin{array}{l}
\bar{x}_{0} \\
\bar{y}_{0} \\
\bar{z}_{0}
\end{array}\right)+\frac{b}{2} \bar{c}\left[\begin{array}{ccc}
1 & 0 & 0 \\
0 & \cos \Gamma & \sin \Gamma \\
0 & -\sin \Gamma & \cos \Gamma
\end{array}\right]\left[\begin{array}{ccc}
\cos i & 0 & -\sin i \\
0 & 1 & 0 \\
\sin i & 0 & \cos i
\end{array}\right]\left(\begin{array}{c}
\left(\psi-\frac{1}{4}\right) \\
0 \\
\left(\frac{t}{c}\right) \bar{\zeta}
\end{array}\right) \\
& =\frac{b}{2}\left(\begin{array}{l}
\bar{x}_{0} \\
\bar{y}_{0} \\
\bar{z}_{0}
\end{array}\right)+ \\
& \frac{b}{2} \bar{c}\left[\begin{array}{ccc}
\cos i & 0 & -\sin i \\
\sin \Gamma \sin i & \cos \Gamma & \sin \Gamma \cos i \\
\cos \Gamma \sin i & -\sin \Gamma & \cos \Gamma \cos i
\end{array}\right]\left(\begin{array}{c}
\left(\psi-\frac{1}{4}\right) \\
0 \\
\left(\frac{t}{c}\right) \bar{\zeta}
\end{array}\right)
\end{aligned}
$$

\section{Subsonic Transport Wing Example}

As an example case, consider a notional subsonic transport wing with a blended winglet (Fig. 4). The wing uses a constant $10 \%$ thick supercritical airfoil and has no twist. The quarter-chord sweep and dihedral are constant from the centerline to the base of the winglet, and the chord exhibits a linear variation from the centerline to approximately $28 \%$ of the semispan, and linear variation from there to the base of the winglet. The combination of linear variations in the chord, sweep, and dihedral out to the tip of the main wing, followed by a dramatic change in the shape of the wing in the winglet, poses a challenge when attempting to fit this wing using spanwise CST functions.

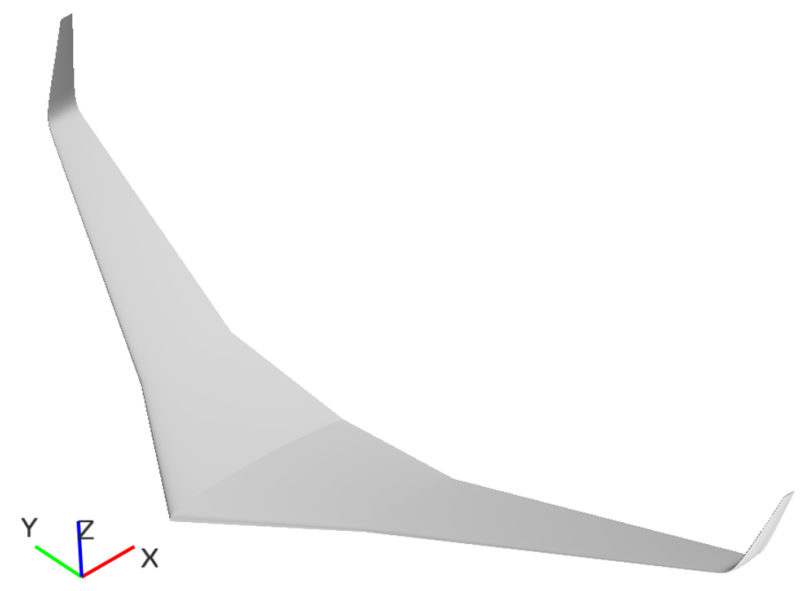

Figure 4. OpenVSP model of a transport wing

The transport wing was modeled in OpenVSP ${ }^{12}$ and exported to a set of discrete points with a grid resolution of 193 cross sections and 101 points per cross section. To test the ability of the different CST parametrization schemes to approximate the surface shape of the transport wing, three types of parametrization were tested for this example: single-section, piecewise, and piecewise with continuity constraints.

\section{A. Single-Section Parametrization}

The first example case attempts to fit the transport wing geometry with a single-section parametrization. Sample CST curve fits for chord and $\mathrm{d} z_{0} / \mathrm{d} s$ are shown in Fig. 5(a). The distribution for chord is linear for the whole wing, including the winglet, whereas the distribution of $\mathrm{d} z_{0} / \mathrm{d} s$ is only linear from the wing root to the tip of the main wing (root of the winglet), at which point it increases dramatically as the winglet curves upward. Consequently, the single-section curve for the chord distribution matches the actual distribution well. On the other hand, a single-section CST curve for $\mathrm{d} z_{0} / \mathrm{d} s$ has difficulty fitting both the constant mainwing section and the greatly-varying winglet section, resulting in a compromise between the two regions and a number of extraneous oscillations in the winglet region.

Figure 6(a) shows contours of fitting error superimposed over the original geometry. At each point on the geometry, the fitting error represents the distance between the original point and the estimated point 


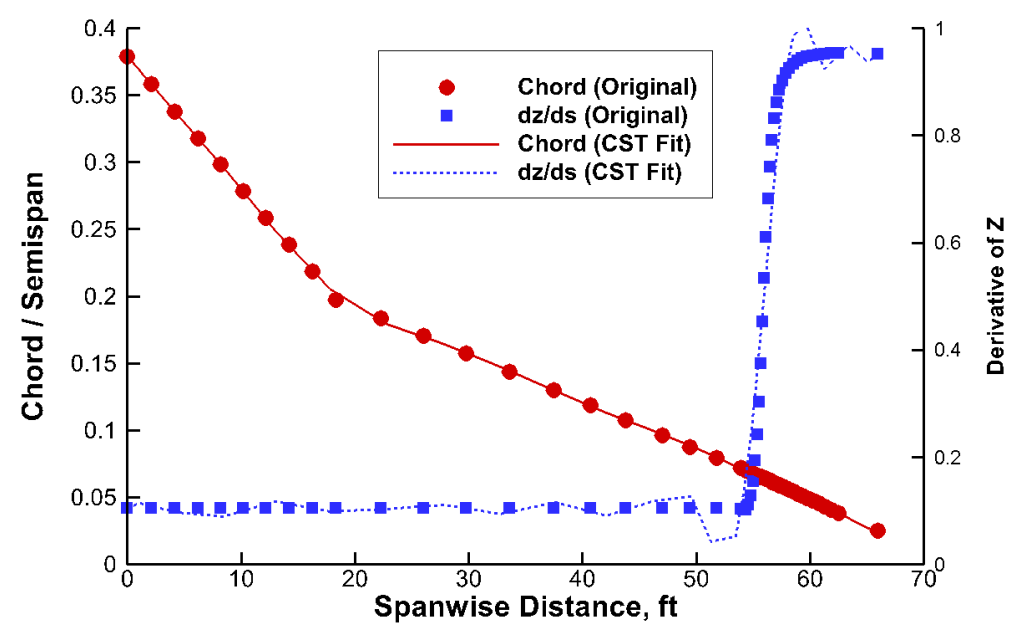

(a) Single-section fit

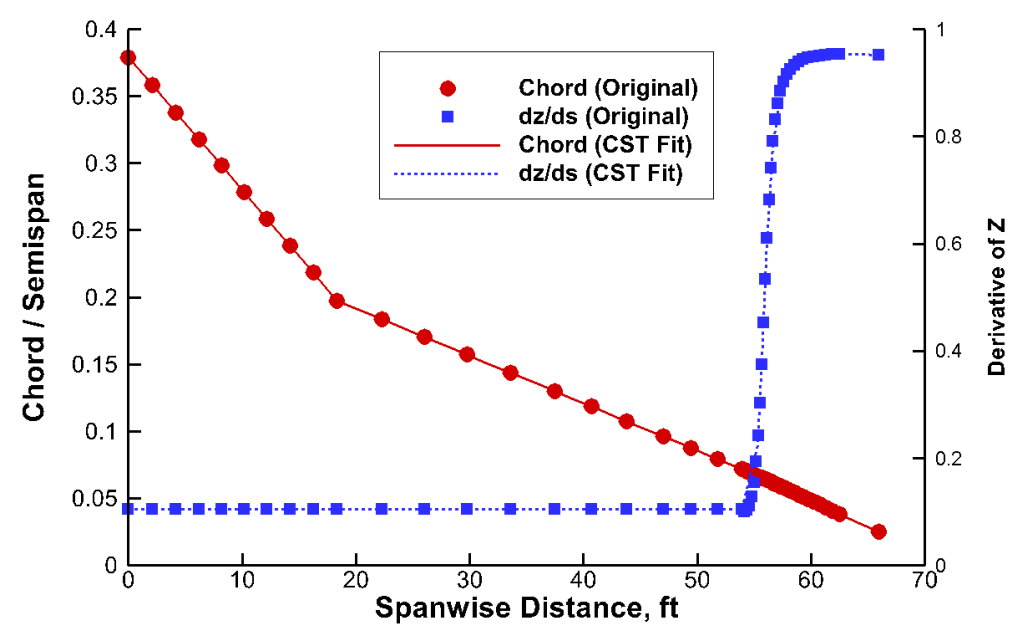

(b) Piecewise fit

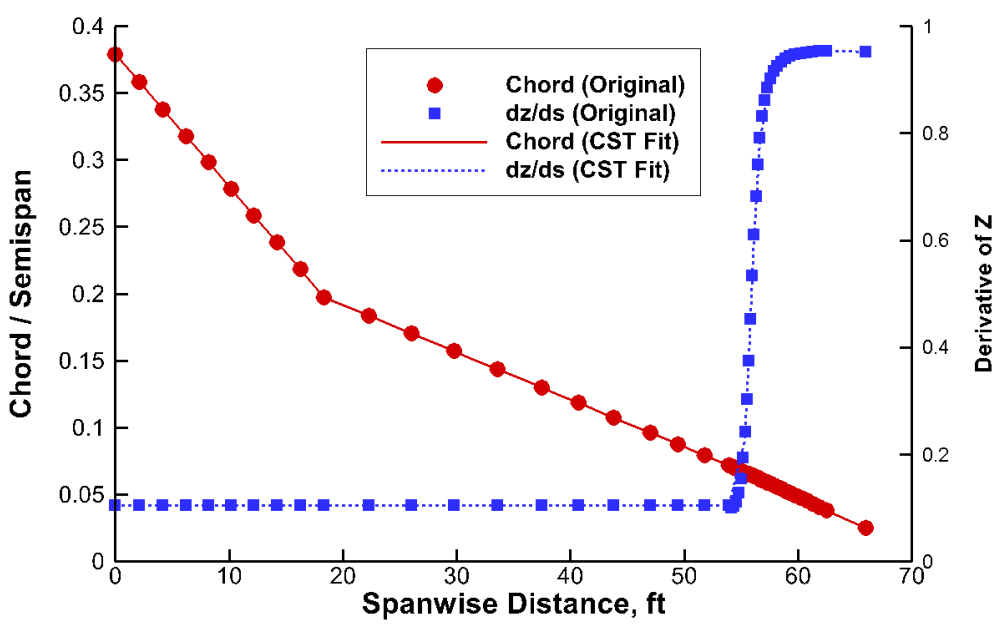

(c) Piecewise fit with continuity constraints

Figure 5. CST curves for chord and $\mathrm{d} z_{0} / \mathrm{d} s$ compared to original transport wing 


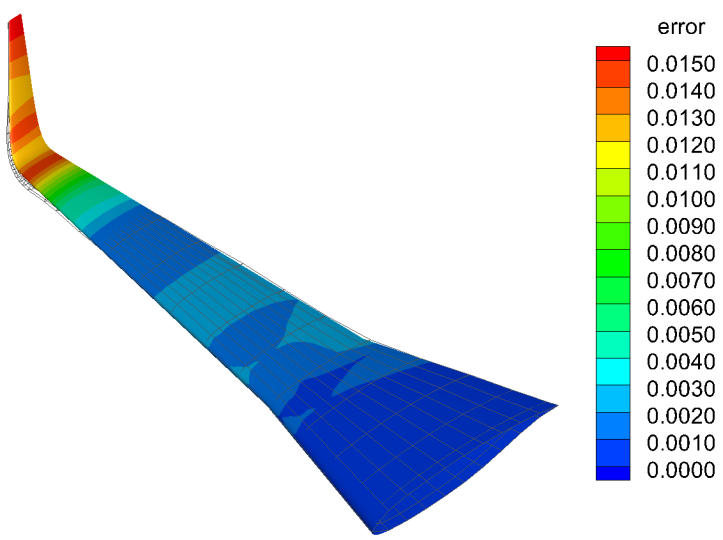

(a) Single-section fit

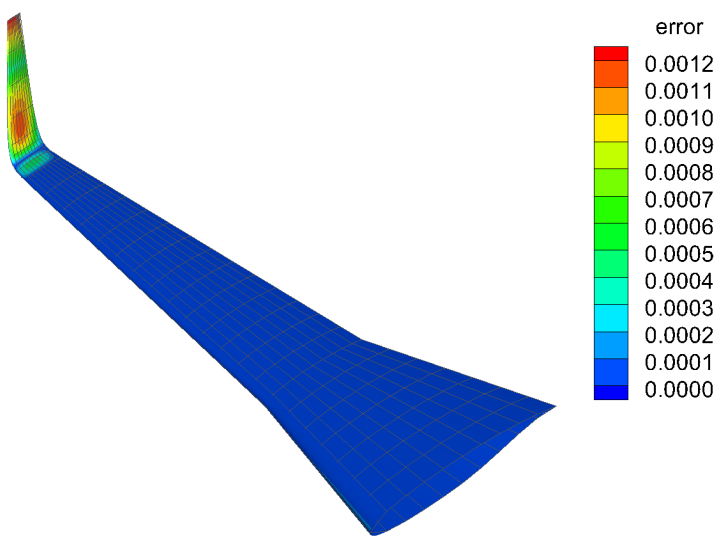

(b) Piecewise fit

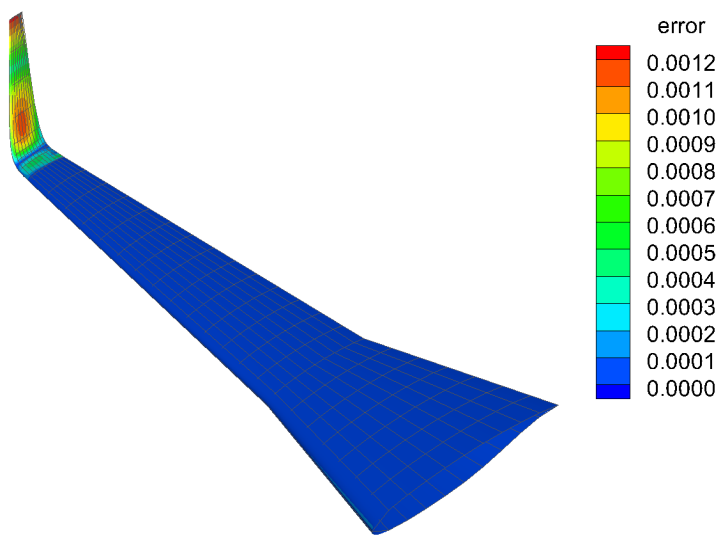

(c) Piecewise fit with continuity constraints

Figure 6. Fitting error (relative to semispan) for single-section CST parametrization of transport wing 
from the CST parametrization at the same fractional spanwise and chordwise location; the error value is expressed as a fraction of the wing semi-span. A skeletal representation of the CST wing is also shown on Fig. 6(a) to illustrate visually where it differs from the original wing surface. The difficulty in matching the $\mathrm{d} z_{0} / \mathrm{d} s$ distribution (and also the $\mathrm{d} y_{0} / \mathrm{d} s$ distribution, not shown) results in fitting errors as high as $1.5 \%$ of the semispan along the winglet surface.

\section{B. Piecewise Parametrization}

The second example uses piecewise parametrization to improve the approximation of the wing surface. This parametrization scheme uses separate curves for the inboard and outboard sections of the main wing on either side of the chord break, plus a third section for the winglet. For this case, only $G^{0}$ continuity is maintained across each of the section boundaries. The plot of spanwise properties (Fig. 5(b)) shows that the use of piecewise equations solves the problem of oscillations in the curve fit seen previously.

Figure 6(b) shows the contours of fitting error plotted on the original surface and a skeletal outline of the surface of the CST wing. Note that the magnitude of the contour scale is smaller by an order of magnitude than the scale in Fig. 6(a), showing that the maximum fitting error is less than one tenth of the maximum error seen in the single-section parametrization.

\section{Piecewise Parametrization with Continuity Constraints}

The final example adds continuity constraints to the piecewise parametrization of the previous example. The additional constraints are $G^{2}$ continuity between the upper and lower surfaces of the airfoil at the nose, plus $G^{1}$ continuity of the reference axis slopes $\left(\mathrm{d} x_{0} / \mathrm{d} s, \mathrm{~d} y_{0} / \mathrm{d} s\right.$, and $\left.\mathrm{d} z_{0} / \mathrm{d} s\right)$ at the root of the winglet, which is in effect a second-order continuity condition because the constraint is imposed on the slope of the reference axis.

As can be seen in Fig. 5(c), the addition of the $G^{1}$ continuity constraint to the CST function for $\mathrm{d} z_{0} / \mathrm{d} s$ makes an imperceptible change to the shape of the function in the vicinity of the winglet root, relative to the unconstrained parametrization. This is because the dihedral changes rapidly in this region and a small initial error is quickly corrected.

Figure 6(c) shows that the additional continuity constraints result in a very small increase in the fitting error at the winglet root. This is due to the necessary compromise between minimizing the approximation error to the actual surface points, and matching the desired slope at the joint exactly. The original OpenVSP model has only zero-order continuity of the dihedral at the winglet root, so imposing first-order continuity slightly degrades the fidelity to the original surface coordinates.

\section{Conclusion}

This paper has laid out a comprehensive method for using three-dimensional CST parametrization to represent aircraft wings, and other lifting surfaces. The parameters chosen are familiar to the aircraft designer for describing wings, and the independent parametrization means that parameters with linear variation (such as chord in the transport wing example) can be represented with only a few values, whereas parameters with highly non-linear variation (such as dihedral in the example) can be represented with a larger number of values. By representing these parameters as a function of spanwise distance $(s)$, rather than of transverse distance $(y)$ as in previous studies, the three-dimensional formulation laid out in this paper is suitable for a wider variety of wing shapes than previous formulations - particularly for wings with significant non-planar components such as blended winglets and box wings. With minor modifications, the methodology could also be used to describe other shapes, such as fuselages and nacelles.

Single-section parametrization can be effective in approximating the surface of a simple trapezoidal wing with modest variations in chord, sweep and dihedral. For more challenging cases, such as the blended winglet example shown here, or for advanced configurations such as a blended wing body, the use of piecewise parametrization can be crucial for accurately describing the surface.

However, the use of piecewise parametrization can introduce discontinuities into what were originally fully smooth and continuous functions, which is a concern that has not been addressed in previous studies. This paper introduces modifications that can be made to the piecewise representation for enforcing continuity between segments. On the one hand, the use of continuity constraints tends to slightly increase the fitting error near the section breaks relative to the unconstrained case; on the other hand, enforcing continuity can 
maintain certain characteristics of the wing that can be essential to its performance. For example, firstor even second-order spanwise continuity of the surface could help maintain laminar flow under conditions of modest spanwise flow. Additionally, second-order continuity at the airfoil leading edge can help reduce drag in off-design conditions. Enforcing continuity constraints can be especially important when the CST parameters are used to modify the wing surface during an optimization process, to stop what was originally a mild discontinuity from becoming a severe one as the optimization proceeds.

\section{Acknowledgments}

This work was conducted as part of the NASA Transformational Tools and Technologies Project, led by James D. Heidmann, within the Multi-Disciplinary Design, Analysis and Optimization element, led by Jeffrey K. Viken. The author wishes to thank Dr. Natalia Alexandrov for her editorial assistance.

\section{References}

\footnotetext{
${ }^{1}$ Kulfan, B. M. and Bussoletti, J. E., "Fundamental' Parametric Geometry Representations for Aircraft Component Shapes," 11th AIAA/ISSMO Multidisciplinary Analysis and Optimization Conference, AIAA, Portsmouth, VA, 2006.

${ }^{2}$ Kulfan, B. M., "A Universal Parametric Geometry Representation Method - CST," 45th AIAA Aerospace Sciences Meeting and Exhibit, AIAA, Reno, NV, 2007.

${ }^{3}$ Kulfan, B. M., "Recent Extensions and Applications of the 'CST' Universal Parametric Geometry Representation Method," 7th AIAA Aviation Technology, Integration and Operations Conference (ATIO), AIAA, Belfast, Northern Ireland, 2007.

${ }^{4}$ Powell, S. and Sóbester, A., "Application-Specific Class Functions for the Kulfan Transformation of Airfoils," 13th AIAA/ISSMO Multidisciplinary Analysis Optimization Conference, AIAA, Fort Worth, TX, 2010.

${ }^{5}$ Albert, M. and Bestle, D., "Automatic Design Evaluation of Nacelle Geometry Using 3D-CFD," 15th AIAA/ISSMO Multidisciplinary Analysis and Optimization Conference, AIAA, Atlanta, GA, 2014.

${ }^{6}$ Morris, C. C., Allison, D. L., Schetz, J. A., Kapania, R. K., and Sultan, C., "Parametric Geometry Model for Design Studies of Tailless Supersonic Aircraft," Journal of Aircraft, Vol. 51, No. 5, 2014, pp. 1455-1466.

${ }^{7}$ Tufts, M. W., Reed, H. L., and Saric, W. S., "Design of an Infinite-Swept-Wing Glove for In-Flight Discrete-RoughnessElement Experiment," Journal of Aircraft, Vol. 51, No. 5, 2014, pp. 1618-1631.

${ }^{8}$ Farouki, R. T., "The Bernstein Polynomial Basis: A Centennial Retrospective," Computer Aided Geometric Design, Vol. 29, No. 6, 2012, pp. 379-419.

${ }^{9}$ Lane, K. A. and Marshall, D. D., "A Surface Parameterization Method for Airfoil Optimization and High Lift 2D Geometries Utilizing the CST Methodology," 47th AIAA Aerospace Sciences Meeting Including the New Horizons Forum and Aerospace Exposition, AIAA, Orlando, FL, 2009.

${ }^{10}$ Barsky, B. A. and DeRose, A. D., "Three Characterizations of Geometric Continuity for Parametric Curves," Tech. Rep. UCB/CSD-88-417, EECS Department, University of California, Berkeley, May 1988.

${ }^{11}$ Marshall, D. D., "Creating Exact Bezier Representations of CST Shapes," 21st AIAA Computational Fluid Dynamics Conference, AIAA, San Diego, CA, 2013.

${ }^{12}$ Hahn, A., "Vehicle Sketch Pad: A Parametric Geometry Modeler for Conceptual Aircraft Design," 48th AIAA Aerospace Sciences Meeting Including the New Horizons Forum and Aerospace Exposition, AIAA, Orlando, FL, 2010.
} 